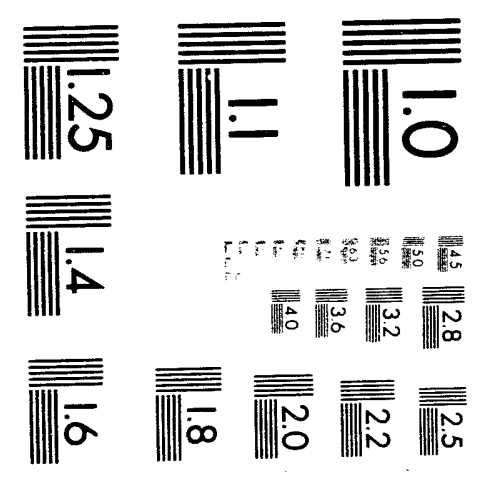



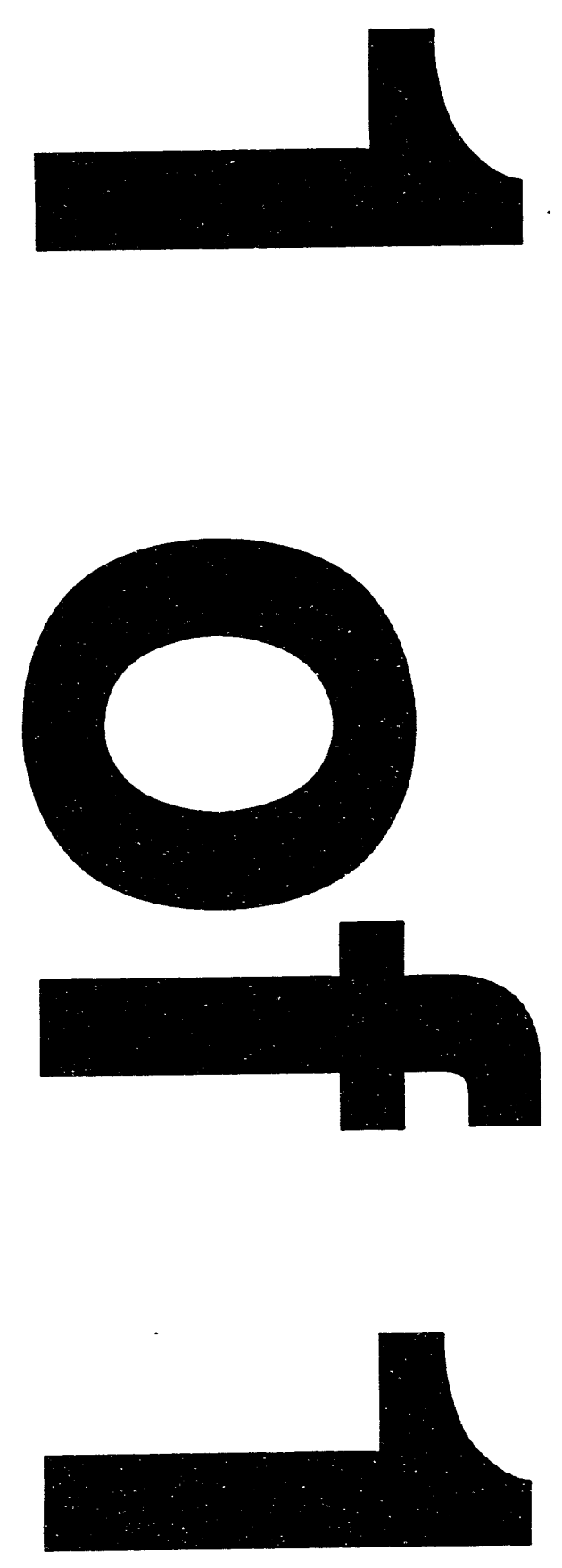


\title{
Measuremenr of Multi-Bunch Transfer Functions Using Time-Domain Data and Fourier Analysis *
}

\author{
by H. Hindi, L. Sapozhnikov, J. Fox, S. Prabhakar, G. Oxoby, I. Linscott \\ Stanford Linear Accelerator Center \\ Stanford University, Stanford, CA 94309 \\ and \\ A. Drago \\ INFN Laboratori Nazionali, Frascati, Italy
}

\begin{abstract}
Multi-bunch transfer functions are principal ingredients in understanding both the behavior of high-current storage rings as well as control of their instabilities. The measurement of transfer functions on a bunch-by-bunch basis is particularly important in the design of active feedback systems. Traditional methods of network analysis that work well in the single bunch case become difficult to implement for many bunches. We have developed a method for obtaining empirical estimates of the multi-bunch longitudinal transfer functions from the time-domain measurements of the bunches' phase oscillations. This method involves recording the response of the bunch of interest to a whitenoise excitation. The transfer function can then be computed as the ratio of the fast Fourier transforms (FFTs) of the response and excitation sequences, averaged over several excitations. The calculation is performed off-line on bunch-phase data and is well-suited to the multi-bunch case. A description of this method and an analysis of its performance is presented with results obtained using the longitudinal quick prototype feedback system developed at SLAC.
\end{abstract}

\section{INTRODUCTION}

Many fundamental methods of system analysis and control theory rely on frequency domain representations of the system of interest. Often the design of control systems is based on transfer function measurements of system elements, in which the complex frequency response of a system is measured from two accessible ports of the system. The design of feedback control systems for accelerators presents many opportunities to apply these principles. A simple system, such as the longitudinal motion of a single-bunch in a storage ring, can be directly measured in the frequency domain using laboratory instruments such as a network analyzer that excites the beam through a driving port (such as an If cavity), and observes the motion of the beam through a measurement port (such as a position-monitor clectrode). However, for a multi-bunch system, traditional two-port network analysis techniques cannot be applied if one desires to measure transfer functions of individual bunches, or cross-coupling functions in which the excitation is applied to bunch $i$ but the response is detected from bunch $j$. Such measurements are extremely useful in the characterization of coupling strengths between bunches and in the determination of the closed-loop frequency response of the complete feedback system.

Many new accelerator and synchrotron light facilities propose to control multi-bunch instabilities through the use of bunch-by-bunch feedback systems $[1,2]$. Such systems calculate correction signals for a bunch from past measurenents of only that bunch. The longitudinal quick prototype developed at SLAC as a component of the PEP-II R\& D program uses digital signal-processing microprocessors (DSPs) to implement a multi-bunch feedback system [3,4,5]. One feature of this implementation is the incorporation of general-purpose, signal-processing computers with availatele

\footnotetext{
*Work supported by Department of Energy contract DE-AC03-76SF00515.
}

Presented at the Beam Instrumentation Workshop, Santa Fe, New Mexico, October 20 23, 1993.

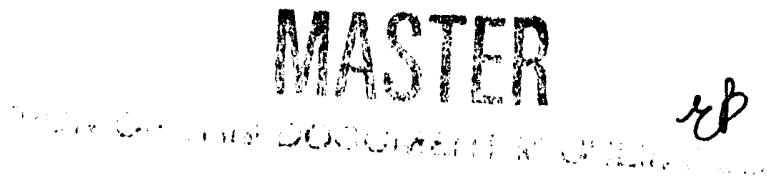


internal memory buffers. With such a processing architecture it is possible to record bunch oscillation data as well as store bunch-excitation sequences in the processor memory. This flexibility allows analysis of time-domain records of the bcam motion, and allows the application of arbitrary excitation patterns to selected bunches. The record and excitation processes can be performed while the feedback system is operational or turned off, and allow multi-bunch transfer functions to be measured with the use of off-line Fourier analysis techniques.

\section{MEASUREMENT FORMALISM}

Suppose we want to analyze a discrete-time system with transfer function (frequency response) $H\left(e^{j \omega}\right)$ and impulse response $h(n), n=1,2, \ldots$. The Discrete-Time Fourier Transform (DTFT) of $h(n)$ is $H\left(e^{j \omega}\right)$, where the DTFT of a discrete-time sequence $x(n)$ is defined as

$$
X\left(e^{j \omega}\right)=\sum_{n=-\infty}^{\infty} x(n) e^{-j \omega n}
$$

Note that since the DTFT is a function of $e^{j \omega}$, it is periodic with period $2 \pi$.

Our problem can be stated as follows: given a finite number of input and output samples $u(n)$ and $y(n)$, we would like to estimate the frequency response $H\left(e^{j \omega}\right)$ of the system. Let the sequence $z(n)$ denote the response of the system to the input sequence $u(n)$. Let $y(n)$ denote our measurements that we assume are corrupted by additive zero-mean white noise $v(n)$. Then we have the following (output error) model [6]:

$$
\begin{aligned}
& z(n)=[h * u](n) \\
& y(n)=z(n)+v(n) .
\end{aligned}
$$

This is shown in Fig. 1. The following theorem from Ljung ([6] p. 25, p. 147) provides an answer to our problem:

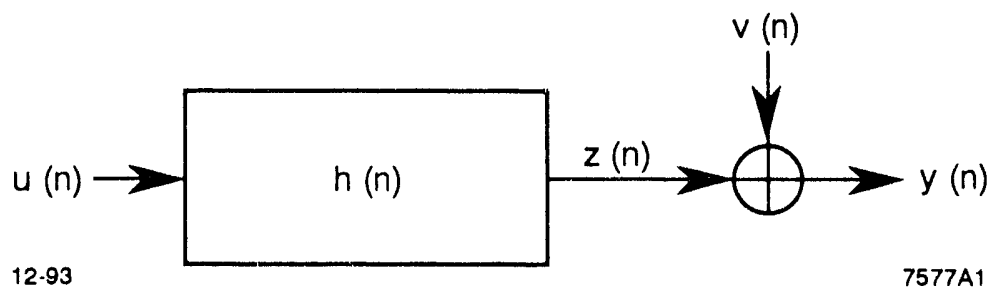

FIG. 1. Block diagram of output error model: a system with impulse response $h(n)$ is excited by an input $u(n)$. The measurements $y(n)$ consist of the system output $z(n)$, corrupted by white noise $v(n)$.

Theorem 1 Let $y(n)$ and $u(n)$ be related by:

$$
y(n)=[h * u](n)+v(n)
$$

where * denotes convolution. Suppose that the input $u(n)$ is unknown for $n<0$ but is bounded. Furthermore let $u(n)$ be periodic with period $N$. Define the $N$-point discrete fourier transform (DFT) of a finite sequence $\{x(n)\}_{0}^{N-1}$ as

$$
X_{N}(k) \triangleq \sum_{n=0}^{N-1} x(n) e^{-i \frac{2 \pi n}{N} k} ; k=0, \ldots, N-1
$$

Then if $U_{N}(k), Y_{N}(k)$ and $V_{N}(k)$ denote the $N$-point DFTs of $u(n), y(n)$ and $v(n)$, respectively, then we have

$$
Y_{N}(k)=H\left(e^{j \frac{2 \pi k}{N}}\right) U_{N}(k)+V_{N}(k)
$$

Thus we can obtain an estimate of $H\left(e^{j \omega}\right)$ at the $N$ points $\omega=\frac{2 \pi k}{N}, k=0,1, \ldots, N-1$ via

$$
\begin{aligned}
\hat{H}\left(e^{j \frac{2 \pi k}{N}}\right) & \triangleq \frac{Y_{N}(k)}{U_{N}(k)} \\
& =H\left(e^{j \frac{2 \pi k}{N}}\right)+\frac{V_{N}(k)}{U_{N}(k)} .
\end{aligned}
$$


Since the noise was assumed to be zero-mean and the input $u(n)$ is known, then by linearity of expectation, the expected value of the error term $V_{N}(k) / U_{N}(k)$ is zero, so $\hat{H}\left(e^{\frac{2 \pi k}{N}}\right)$ is an unbiased estimator of $H\left(e^{j \frac{2 \pi k}{N}}\right)$. This means that this error can be reduced by averaging several independant estimates of $\hat{H}\left(e^{j \frac{2 \pi k}{N}}\right) . \hat{H}\left(e^{j \frac{2 \pi k}{N}}\right)$ is called the enupirical transfer function estimate (ETFE) of $H\left(e^{j \frac{2 \pi k}{N}}\right)$.

In practice, this technique of obtainitu ransfer function estimates using a known periodic excitation has proven very useful because it gives a very quick estimate of the entire spectrum in one pass (sampled at the frequency intervals $1 / \mathrm{N}$ times the sampling frequency). In noisy situations, the theorem above shows that repeated averaging can be used to reduce the noise.

For example, consider a single-bunch beam in a machine such as ALS that has a longitudinal oscillation frequency of about $10 \mathrm{kHz}$. If the longitudinal motion is sampled at $50 \mathrm{kHz}$, then applying eight sequences of a $1024-p o i n t$ white-noise excitation to the system takes about $200 \mathrm{~ms}$. The computations required to calculate the ETFE from the input and output sequences can be performed on an IBM PC in a matter of seconds. Furthermore, the availability of the time-domain sequences makes it possible to use parametric estimation techniques to obtain the actual cocficients of the frequency response. Having an analytic expression for the frequency response is very important from the control systems point of view, as the model of the system determines what transfer function to use in the feedback.

\section{EMPIRICAL TRANSFER FUNCTION ESTIMATES VIA KNOWN EXCITATION}

In this section we illustrate the effects of the choice of the input excitation on the ETFE via a realistic computer simulation. This computer simulation allows us to plot the signals at all points in our system, even inside the digital parts.

Consider analyzing the system shown in Fig. 2 using the method above. The figure shows the discrete transfer function of a one-bunch bearn with a synchrotron frequency of $10 \mathrm{kHz}$, sampled at $50 \mathrm{kHz}$ as in the example above. The instrument uses 8 -bit $\mathrm{A} / \mathrm{D}$ and $\mathrm{D} / \mathrm{A}$ converters with a range of $\pm 2.5 \mathrm{~V}$. The output is corrupted by white noise of $20-m \mathrm{rms}$.
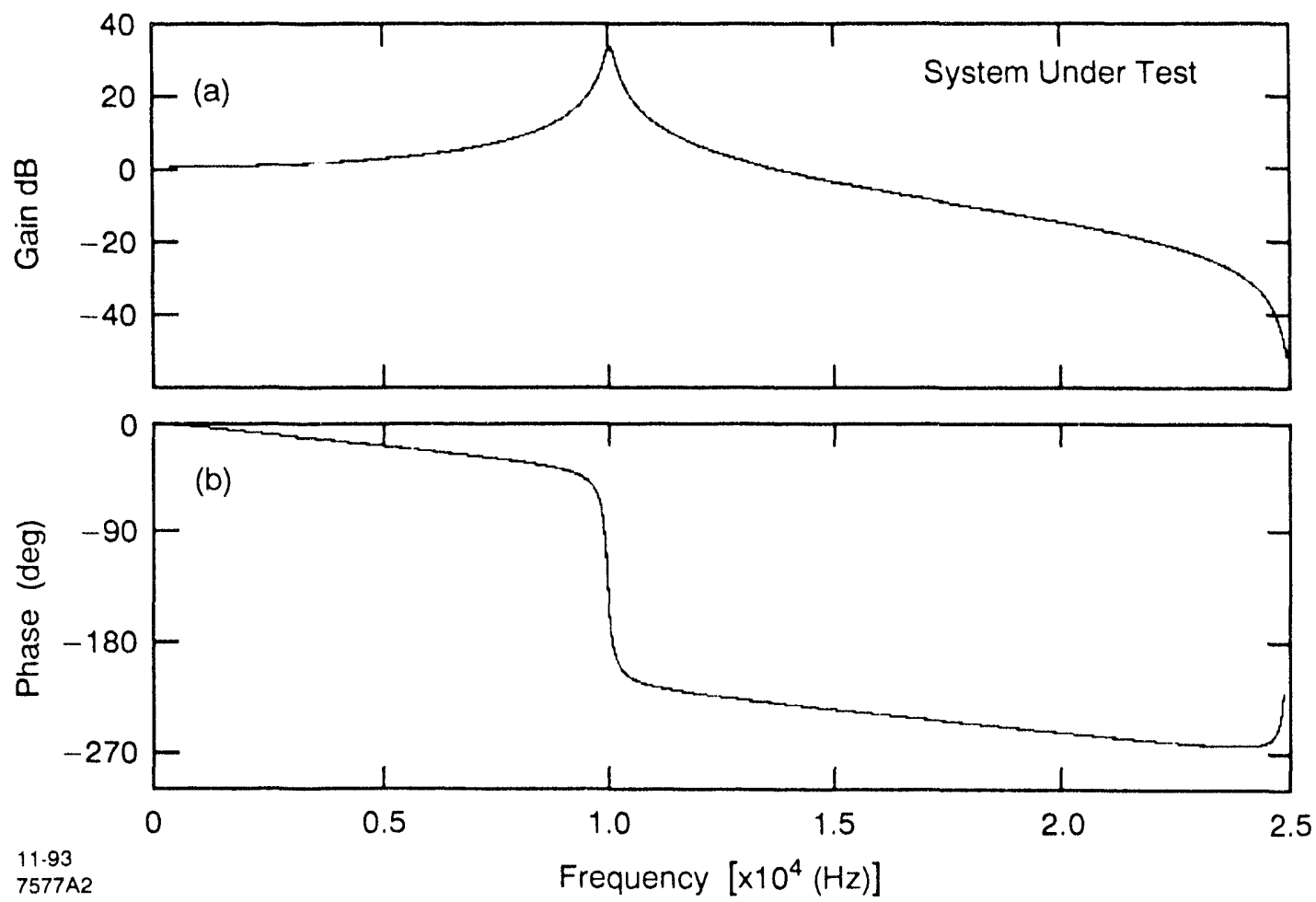

FIG. 2. Frequency reponse of a single bunch with a $10 \mathrm{khz}$ synchrotron frequency. 


\section{A. Empirical Transfer Function Estimates via White Noise Excitation}

We choose $u(n)$ to be a periodic white-noise sequence with period $N=1024$. White noise is a traditional choice for $u(n)$ since it provides a broadband excitation for the unknown system. Four periods of $u(n)$ are applied to the system and four 1024-point output sequences $y(n)$ are recorded. The DFTs are computed off line using the FFT algorithm. The transfer function is then computed as the average of the four independent $\hat{H}\left(e^{j \frac{2 \pi k}{N}}\right)$ estimates from each output sequence.

Figure 3 shows the time history and the power spectrums of the quantized 1024-point white-noise input of $0.12 \mathrm{~V}$ rms and the digitized output. Since the system response is large at resonance we can only drive the system with a few counts from the output D/A before the input A/D converter saturates. Also, note that the input spectrum is almost flat, while the dc-to-peak range of our output is $60 \mathrm{~dB}$. Thus, the experiment clearly has not made the best use of our input and output dynamic ranges. Nevertheless, it was still possible to compute a fairly reasonable-looking ETFE using four averages (see Fig. 4). 

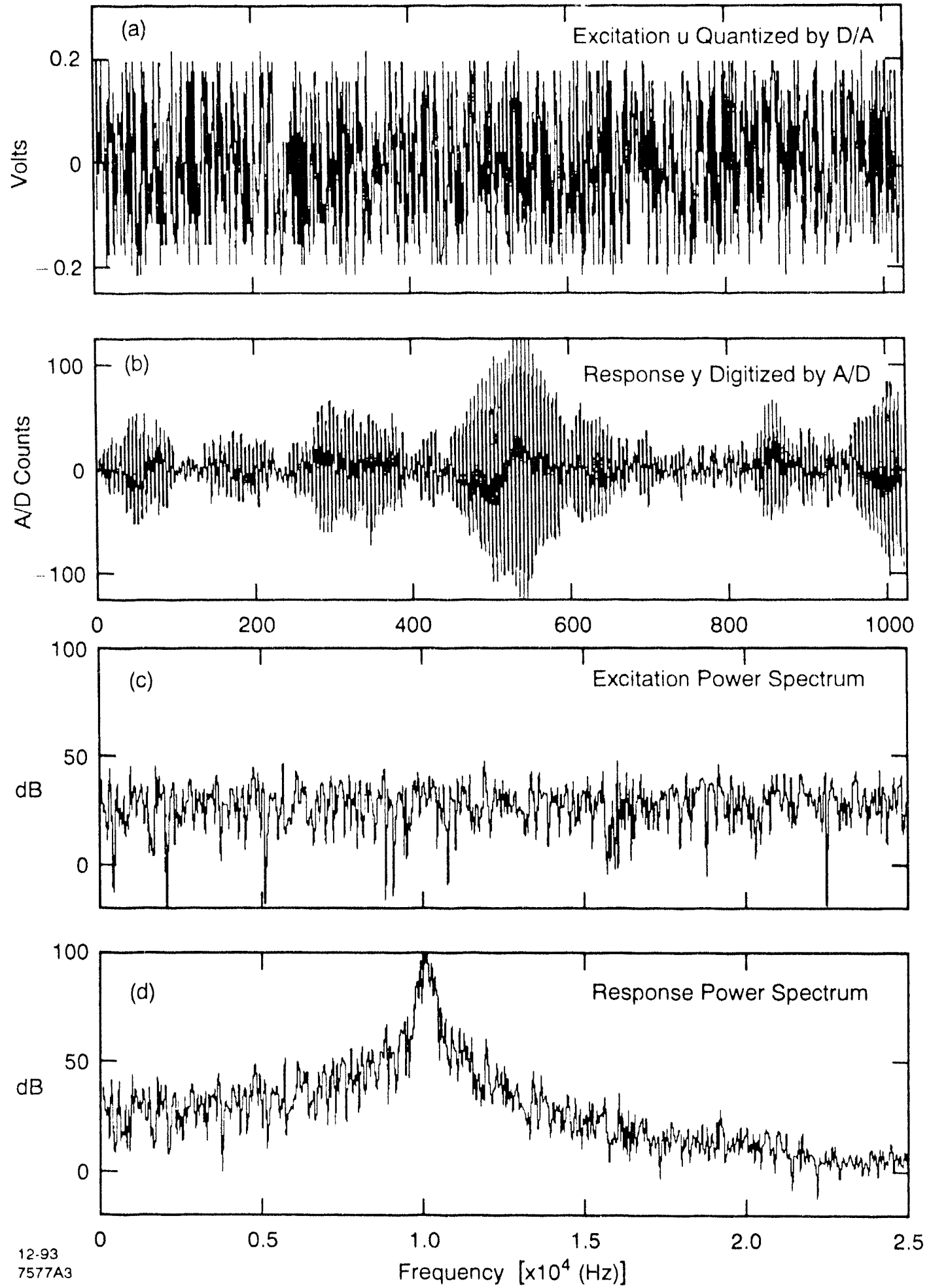

FIG. 3. Time sequences of the excitation (3a), the system response (3b), the associated excitation power spectrum (3x). and the response power spectrum (3d). 

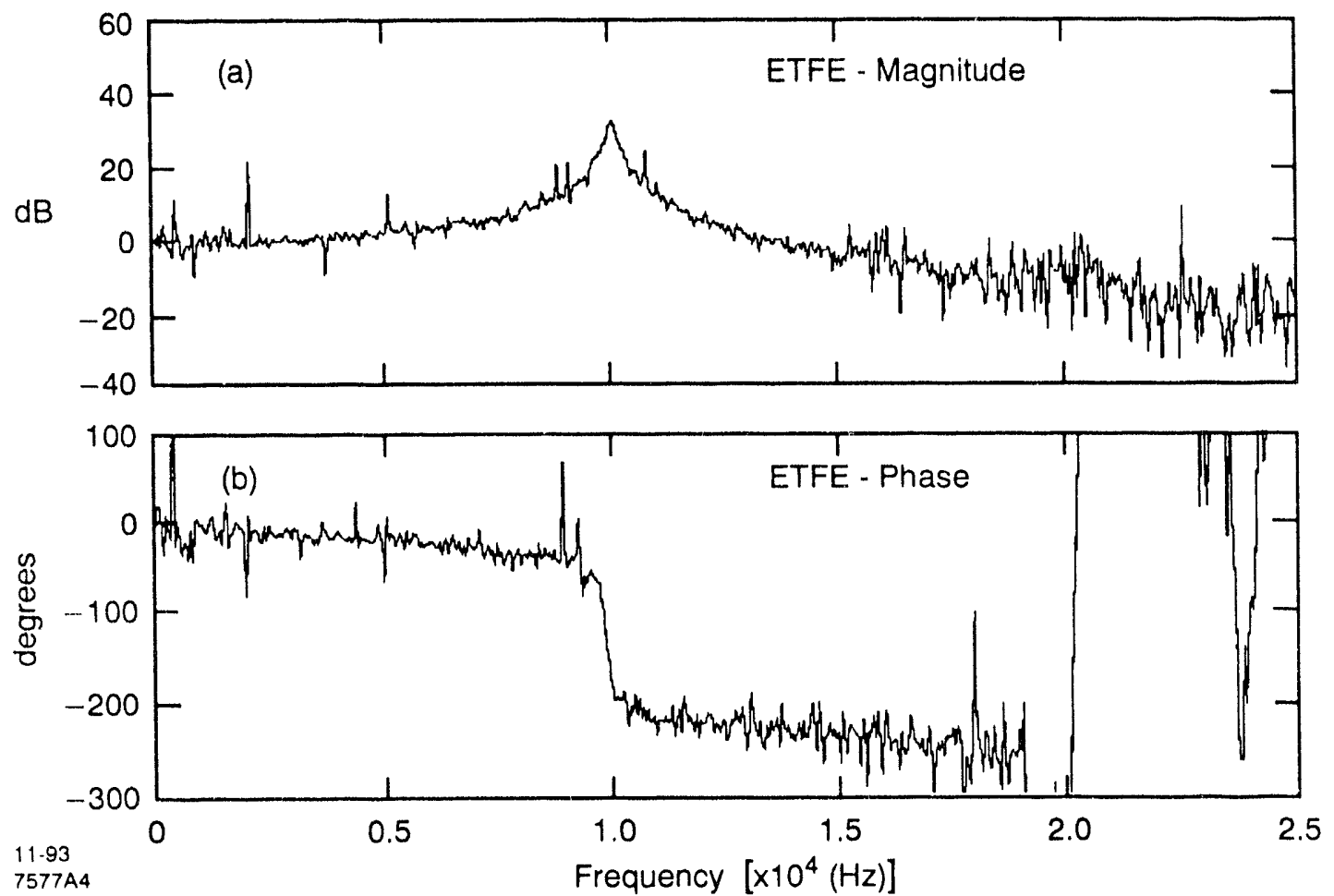

FIG. 4. Empirical transfer function estimate (ETFE) of the system of Fig. 2, computed from time sequences $3 a$ and $3 \mathrm{~b}$ via the ratio of their Fourier transforms and averaged over four excitations.

\section{B. Empirical Transfer Function Estimates via Shaped Excitation}

Despite its crude appearance, Fig. 4 contains valuable information about the system. We will now outline an iterative procedure that exploits the information contained in this ETFE in a direct way and uses it to construct a spectrally shaped input-excitation sequence. Such a spectrally shaped excitation uses information available about the test system to distribute the dynamic range requirements more evenly between the input and output of the measuring instrument [7]. In essence, one should drive the system hard at frequencies where it has little response, and be gentle in frequency ranges where the system responds strongly. Alternatively, if the system parameters are well known one can create the shaped excitation directly from knowledge of the system-transfer functions.

Given an initial white-noise ETFE, the custom input, $u_{c}$, is obtained as follows [7]:

1. Form the lower half of the custom input FFT from the ETFE as

$$
U_{1}\left(e^{j 2 \pi n / N}\right)=\left(\sqrt{\left|\hat{H}\left(e^{j 2 \pi n / N}\right)\right|}\right)^{-1} e^{j \phi_{n}} \quad n=0,1, \ldots, N / 2,
$$

where

$$
\phi_{n}=\left\{\begin{array}{ll}
\operatorname{uniform}(0,2 \pi) & n=1,2, \ldots, N / 2-1 \\
\operatorname{rand}\{-1,1\} & n=0, N / 2
\end{array} .\right.
$$

2. Form the upper half of the input DFT from the lower half via complex conjugation

$$
U_{2}\left(e^{j 2 \pi n / N}\right)=U_{1}\left(e^{j 2 \pi(N-n) / N}\right)^{*} \quad n=N / 2+1, \ldots . N-1 .
$$

3. Form the overall input FFT $U_{c}\left(e^{j 2 \pi n / N}\right)$ as the concatanation of $U_{1}$ and $U_{2}$.

4. Obtain the custom input $u_{c}(n)$ by taking the inverse FFT of $U_{c}\left(e^{j 2 \pi n / N}\right)$. 

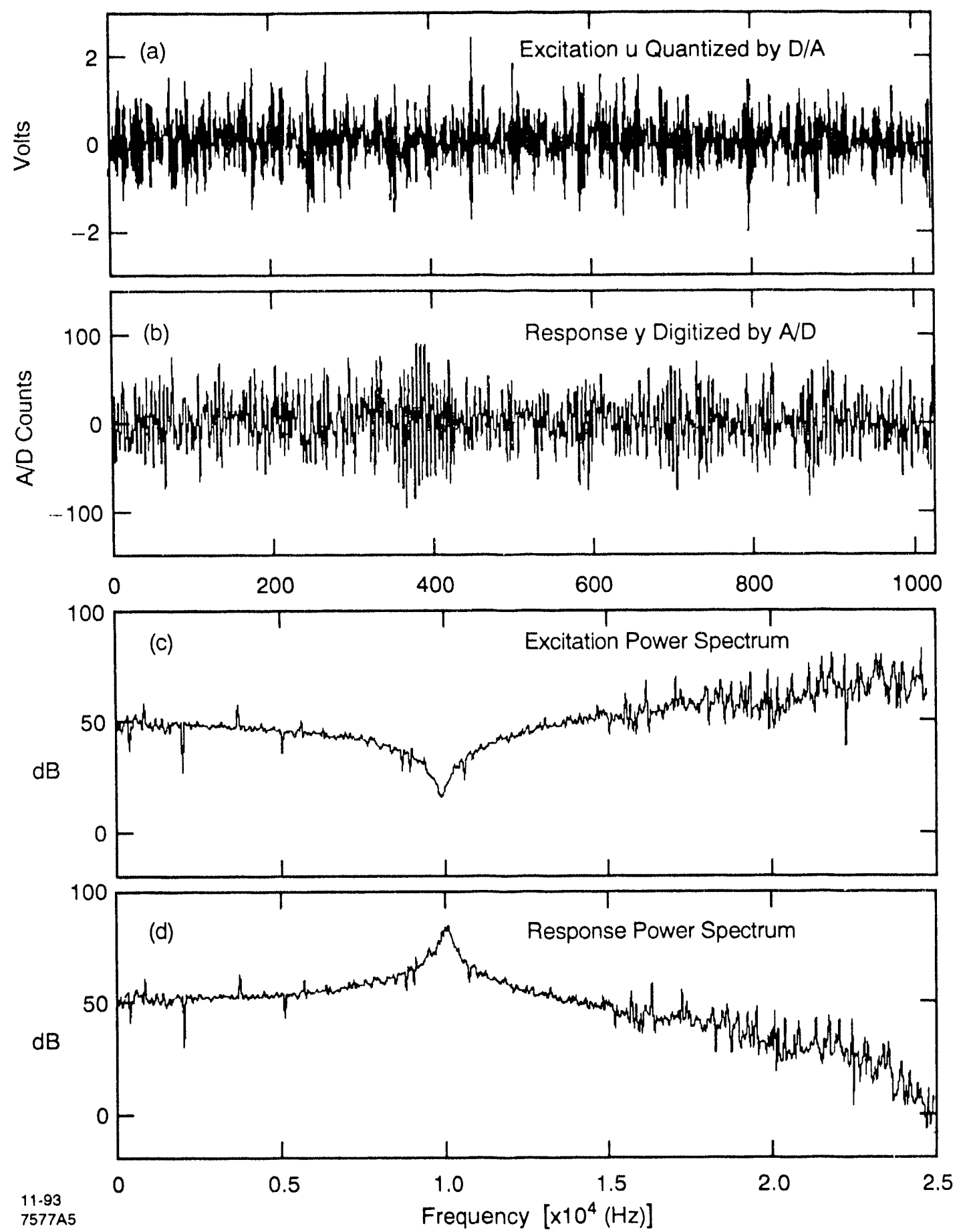

FIG. 5. Time sequences of the shaped excitation (5a), the system response (5b), the associated excitation power spectrum (5c), and the response power spectrum (5d). The shaped excitation shares the system dynamic range between excitation anr! response signals.

The sequence $u_{c}(n)$ can now be used as the periodic excitation sequence and the ETFE can be computed from this as before. Since this input sequence does not have much power at the frequencies of high response in the trist systrm. we can scale this imput to a larger rms value of $0.6 \mathrm{~V} \mathrm{rms,} \mathrm{without} \mathrm{saturating} \mathrm{the} \mathrm{instrument} \mathrm{A/D.} \mathrm{Figure} 5$ shows the time history and the power spectrums of the quantized 1024-point custom input and the digitized system response. The finer resolution at the input reduces the error due to quantization while the signal-to-noise ratio at the ontput is 
maintained, since the excursions are roughly the same as before. The resulting ETFE (four times averaged) is shown in Fig. 6. Note the improved resolution at the notch at high frequencies. This improvement is expected since we increased the relative power of the input at those frequencies.
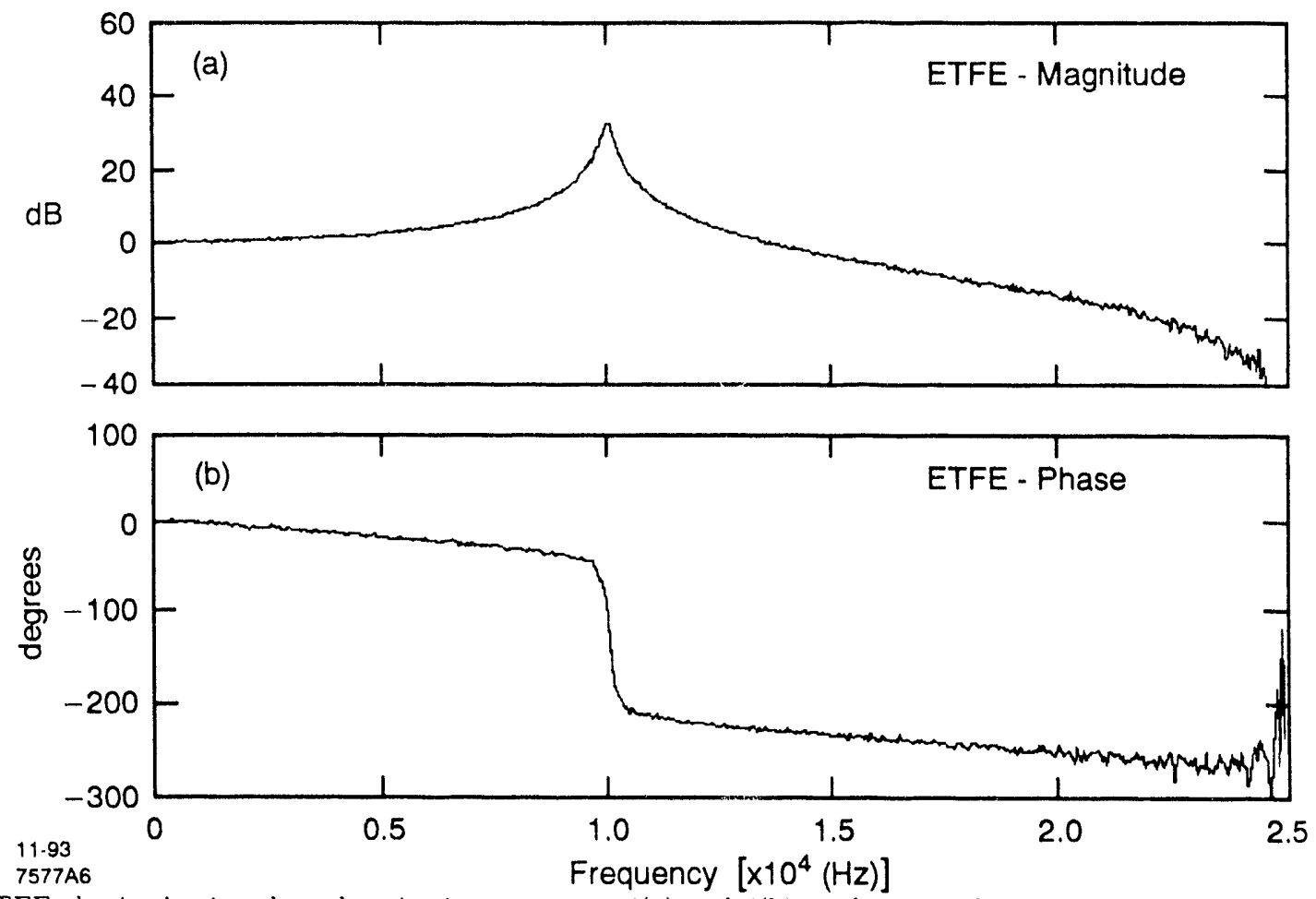

FIG. 6. ETFE obtained using shaped excitation sequences $5(\mathrm{a})$ and $5(\mathrm{~b})$, and averaged over four excitations. The measurement dynamic range now spans $60 \mathrm{~dB}$.

\section{IMPLEMENTATION AND RESULTS}

The essential system features needed to compute the estimated transfer functions are: a means of generating the excitation functions, applying the excitation functions to selected bunches, and recording the system response. Figure 7 shows a block diagram of the signal-processing components of the longitudinal quick prototype [2,4]. The excitation functions $u(n)$ are computed off line (using a high-level language such as Matlab (8]) and transferred to DSP memory with the control programs via the JTAG control link. An external trigger can begin the time-domain excitation or the excitation can start under program control. The system-response records are stored in the DSP general-purpose memory. After a record is recorded, the DSP development system is used to transfer the time records for off-line analysis.

As the DSP memories are 16 kbytes long, it is possible to use excitation sequence lengths of 1024 to 4096 samples. For the ALS operating conditions (revolution frequency of $1.5 \mathrm{MHz}$ ) and a downsampling factor of 24 a 1024 -point record allows frequency resolution of roughly $50 \mathrm{~Hz}$ per computed spectral bin. The software process that excites the beam can run in conjuction with the feedback control program by adding the excitation signal to the computed output signal for bunches under study. Similarly, the control program must save the time record of selected bunch samples as it computes correction signals. These functions add an execution penalty to the software overhead in the DSP when these diagnostic program modes are loaded that is roughly 20 percent of the nominal filter-execution time per bunch.

We implemented the techniques above with the prototype DSP feedback system. Figure 8 shows a transfer function obtained using the prototype system and the ETFE method using a white-noise excitation. For this measurement a 1024-point time record was used, with four excitations averaged. This measurement is of an analog computer that mimics the behavior of a single bunch (an active filter that models the second-order, low-pass properties of the stored bunch) with nominal ALS parameters. (Actual ALS beam measurements are not possible until the completion of the installation of the longitudinal kicker and power amplifier in fall 1993.) 
The estimated transfer function reveals the limited dynamic range of the feedback system input and output stages, which are implemented with 8-bit. $A / D$ and D/A components. This limit restricts the excitation and time-record dynamic range to a maximum of $48 \mathrm{~dB}$ if the signals are exactly matched to the converter's operating ranges. We see that the transfer function measurement in Fig. 8 becomes noisy for frequencies that are roughly 40 dB below the peak response as we would expect. Averaging more excitation/response data sets cannot improve on the signal-to-noise ratio when the signals fall below the quantization limit.

Figure 9 shows a measurement of the transfer function of the same bunch simulator, but using a shaped excitation computed from the ETFE of Fig. 8. The reduced power of the excitation sequence in the region of the central resonance peak allows improvement of the dynamic range of the ETFE measurement as expected.

\section{SUMMARY}

The time-domain processing scheme used in a bunch-by-bunch feedback processing system has been show'n to be capable of measuring transfer functions through the use of Fourier analysis methods. These transfer function measurements may be made on systems for which two-port traditional network methods cannot be applied, and directly allow the coupling between bunches in an accelerator to be measured.

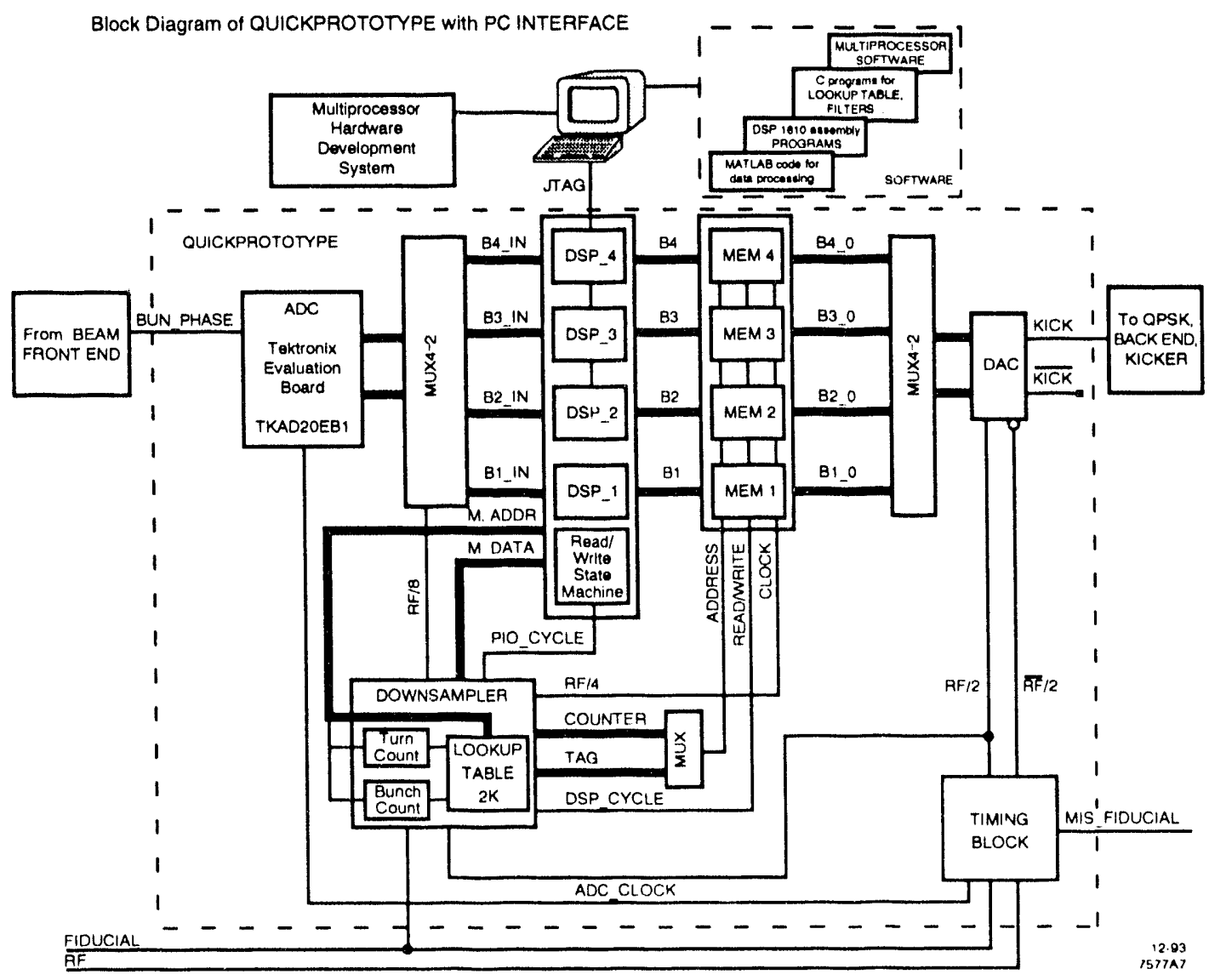

FIG. 7. Block diagram of the digital signal-processing functions of the longitudinal quick prototype. General-purpose [DSP microprocessors are used to provide time excitation functions and to record the system response. 


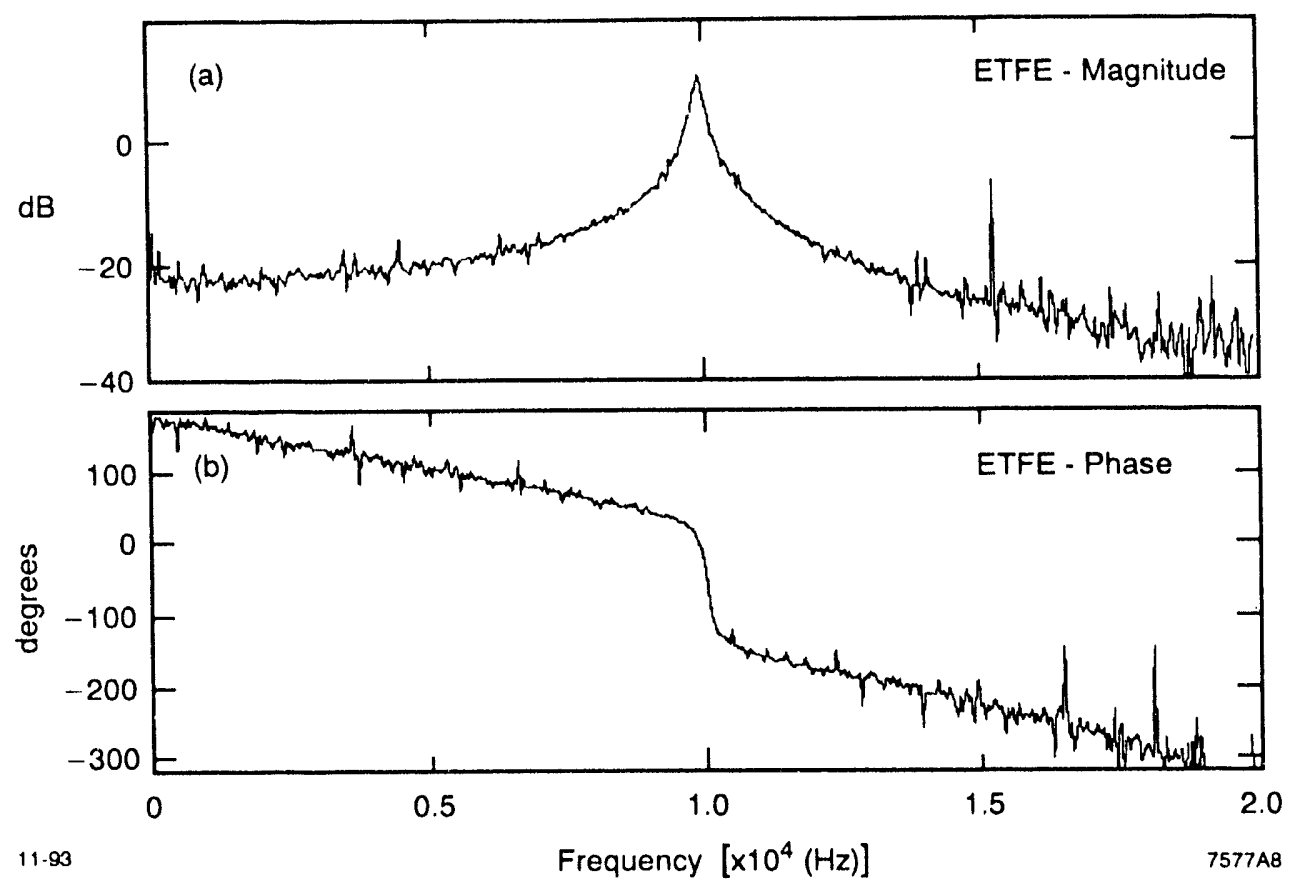

FIG. 8. Laboratory EJTFF measurement of a single-bunch transfer function (second-order active filter) obtained using the quick prototype-fecdback system and a white noise (random) excitation sequence. Four responses are averaged. Note that the dynamic range of the transfer function is limited to roughly $40 \mathrm{~dB}$.

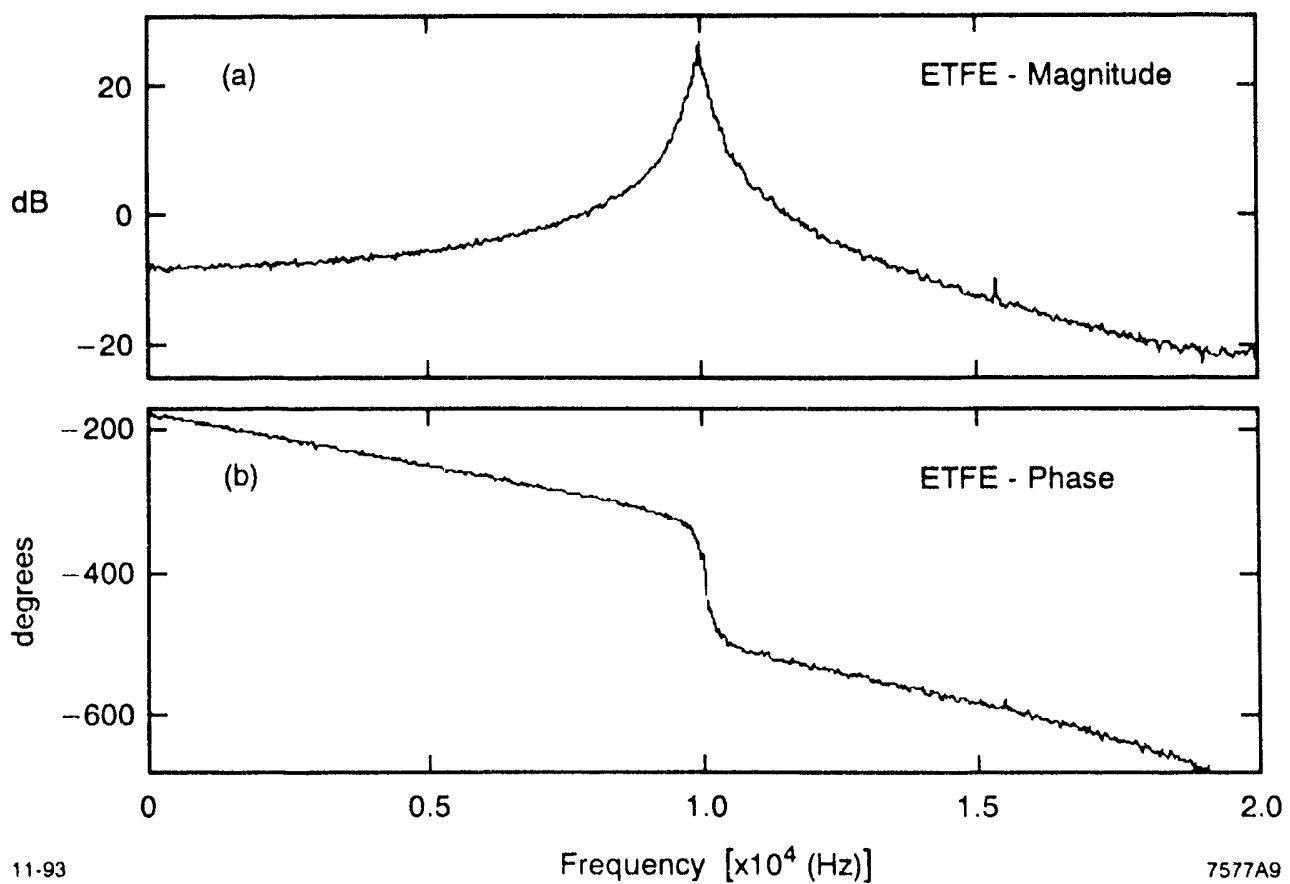

FIG. 9. Laberatory ETFE measurement of a single-bunch transfer function obtained using the quick prototype and a spectrally shaped excitation sequence. The excitation function was computed from the measured system response in Fir. 8 . Four excitations are averaged. The measurement dynamic range now exceeds $60 \mathrm{~dB}$ and noise in the F'TFE is reduced from that seen in Fig. 8. 


\section{REFERENCES}

(1) J. N. Galayda, "Feedback Control of Multi-Bunch Instabilities," The Physics of Particle Accelerators, AlP Conference Proceedings No. 249, p. 663, 1992.

[2] J. Fox, et al. "Feedhack Control of Coupled-Bunch Instabilities," Procceedings of the 1993 Particle Arcelerator" Conference, Washington, DC 1993.

[3] "PEP-Il, An Asymmetric B Factory De... Design Update," Conceptual Design Report Update, SLAC, 1992.

[4] L. Sapozhnikov, et al., "A Longitudinal Multi-Bunch Feedback System Using Parallel Digital-Signal Processors." Proceedings of the 1993 Beam Instrumentation Workshop, Santa Fe, NM 1993.

(5) F. Pedersen, "Multi-Bunch Feedhark Transverse. Longitudinal and RF Cavity Feedback," Proceedings of the 1992 Facturies with e+/e- Rings Workshop. Benalmaldena, Spain. November 1992.

[6] L. Ljung, System Identification: Theory for the User, Prentice-Hall, Englewood Cliffs, N.J 1987.

[7] "Techniques for Signal and Symstem Analysis," Tektronix Signal Analysis Unit, Tektronix Corporation, PX $363010001,1991$.

[8] "MATLAB Reference Guide," The Math Works. Inc., Natick MA. 
